\title{
Complex Morphology Formation in Electrospinning of Binary and Ternary Poly(lactic acid) Solutions
}

\author{
Ehsan Rezabeigi, ${ }^{\dagger}{ }^{\dagger}$ Paula M. Wood-Adams, ${ }^{\ddagger}{ }^{\circledR 0}$ and Nicole R. Demarquette ${ }^{\dagger}$ \\ †Département de Génie Mécanique, École de Technologie Supérieure, 1100 rue Notre-Dame Ouest, H3C 1K3 Montréal, Canada \\ ${ }^{\ddagger}$ Department of Mechanical, Industrial and Aerospace Engineering, Concordia University, 1550 de Maisonneuve Blvd W, H3G 2J2 \\ Montréal, Canada
}

Supporting Information

\begin{abstract}
Electrospinning of ternary systems containing a nonsolvent is of interest because it can produce fibers that are highly porous on the surface and in the core. Despite the growing interest and applications, electrospinning of such ternary systems is challenging due to their evolving viscoelastic properties prior to electrospinning. In this study, we produce porous/rough poly(lactic acid) (PLA) fibers via electrospinning of binary and ternary systems of PLA, dichloromethane (solvent) and hexane (nonsolvent). We introduce a rheological approach to determine the appropriate time for electrospinning of ternary systems which is within at most $10 \%$ of their induction time of crystallization, when incipient

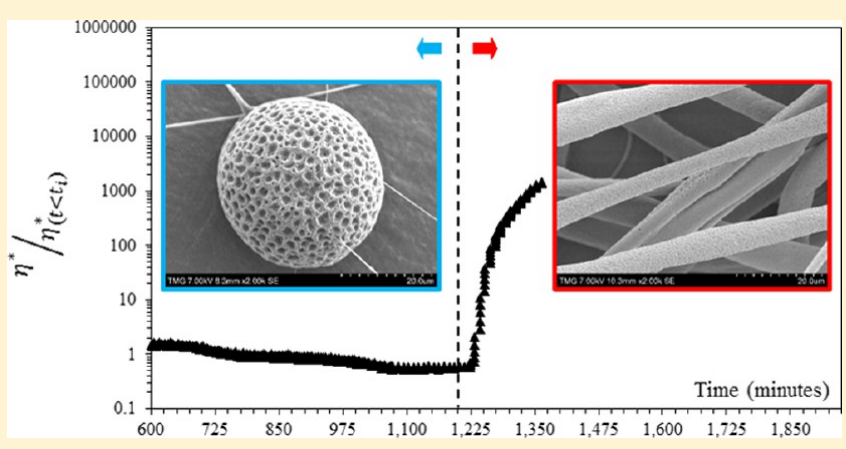
crystals are formed within the polymer-rich phase. These incipient crystals act as nucleators for further crystallization during the process leading to higher crystallinity and more $\alpha$ form crystals. Surprisingly, the pore morphology and crystallinity of the aselectrospun fibers are drastically changed after a room temperature solvent exchange. During this process, cold crystallization and $\alpha^{\prime}$ to $\alpha$ recrystallization occur for all systems. Also, the surface porosity of the fibers is decreased after the solvent exchange, except for the fibers produced from the ternary systems with higher PLA concentrations. Our results indicate that the crystallinity and surface texture of electrospun membranes and thus their performance may change during subsequent analysis and in-service applications, even when a highly volatile solvent is used as a spinning medium.
\end{abstract}

\section{INTRODUCTION}

Fibrous membranes produced by electrospinning of polymer melts or solutions have gained interest and applications in various fields from filtration and sensors to tissue engineering and drug delivery. ${ }^{1-7}$ This technique can be used to produce polymeric membranes with narrow pore size distributions, a high degree of interconnectivity, and low tortuosity, consisting of microsized or nanosized fibers. ${ }^{1,3,8-10}$ There are several processing, material, and ambient parameters affecting these characteristics. This makes electrospinning a flexible fabrication technique allowing for the design of membranes with specific characteristics suitable for target applications. ${ }^{1,3,6,11,12}$ It also makes the process particularly complex to understand from a fundamental point of view.

The crystallinity and surface texture and porosity of the electrospun fibers significantly influence their performances. $3,8,13,14$ Surface porosity of the fibers is also essential for applications requiring deposition of nanoparticles, drugs, and enzymes. ${ }^{1,2,8}$ Furthermore, mechanical and physical properties and permeability of the electrospun membranes depend on their crystallinity. ${ }^{9,13,15}$ A good understanding of the pore formation and crystallization process during electrospinning helps to better design the fabrication of membranes with desirable characteristics for specific applications.
There are many methods to create porosity and roughness on the fibers, for example, salt-induced electrospinning or electrospinning of polymer blends. However, such techniques using a porogen require post-treatments to selectively eliminate the salt or sacrificial polymer(s). ${ }^{2,5,16}$ The main mechanisms that are involved in pore formation during electrospinning of a polymer solution include breath figures, thermally induced phase separation (TIPS), and humidity-induced phase separation. ${ }^{1,3,8,9,16}$ The rapid evaporation of a volatile solvent from a polymer solution during electrospinning can cause thermodynamic instability in the system which is the driving force for phase separation of the solution into polymer-rich and polymer-lean. ${ }^{1,17-21}$ Because of this rapid solvent evaporation and the significant increase in surface area ${ }^{1,6}$ of the system, the surface temperature of the jet drops during electrospinning. ${ }^{1,8,16}$ This sudden decrease in temperature can result in TIPS as well as condensation of humidity as tiny water droplets on the surface of the jet. The subsequent evaporation of these water droplets contributes to pore formation by leaving imprints on the surface of the fibers (known as breath figures).

Received: January 14, 2018

Revised: $\quad$ March 30, 2018 
Table 1. Chemical Structures and the Physical and Chemical Properties of the Materials Used in This Study ${ }^{11,16,17}$

\begin{tabular}{|c|c|c|c|c|c|}
\hline \multirow{2}{*}{ Chemical } & \multirow{2}{*}{ Chemical structure } & \multicolumn{2}{|c|}{ Volatility } & \multirow{2}{*}{$\begin{array}{l}\text { Density } \\
\left(\mathrm{g} / \mathrm{cm}^{3}\right)\end{array}$} & \multirow{2}{*}{$\begin{array}{c}\text { Molecular } \\
\text { weight } \\
(\mathrm{g} / \mathrm{mol})\end{array}$} \\
\hline & & $\begin{array}{c}\text { Boiling } \\
\text { point }\left({ }^{\circ} \mathrm{C}\right)\end{array}$ & $\begin{array}{c}\text { Vapor pressure } \\
(\mathrm{mmHg})\end{array}$ & & \\
\hline PLA & & - & - & $1.24^{[\mathrm{a}]}$ & $72.06^{[b]}$ \\
\hline DCM & & $40^{[c]}$ & $376\left(\text { at } 21^{\circ} \mathrm{C}\right)^{[\mathrm{c}]}$ & $1.33^{[\mathrm{c}]}$ & 84.93 \\
\hline Hexane & & $69^{[\mathrm{c}]}$ & $128\left(\text { at } 21^{\circ} \mathrm{C}\right)^{[c]}$ & $0.65^{[\mathrm{c}]}$ & 86.17 \\
\hline Methanol & & $64.7^{\text {[c] }}$ & $97.7\left(\text { at } 20^{\circ} \mathrm{C}\right)^{[\mathrm{c}]}$ & $0.79^{[\mathrm{c}]}$ & 32.04 \\
\hline
\end{tabular}

${ }^{a}$ The density of PLA depends on its degree of crystallinity. ${ }^{18,19}$ The value $1.24 \mathrm{~g} / \mathrm{cm}^{3}$ indicated here is the density reported by the supplier for the asreceived PLA. ${ }^{b}$ This is the mer molecular weight of PLA. ${ }^{c}$ Specified by the supplier.

Furthermore, if water is a nonsolvent for the polymer and is miscible with the solvent, these droplets can also diffuse into the solution and contribute to the pore formation by promoting phase separation. ${ }^{1,8,16,18,20,22}$ Note that the pores formed as a result of breath figures are normally described as uniformly circular which are densely packed and similar in size and depth. ${ }^{1,16}$ However, depending on the system and electrospinning parameters, some irregularity such as elongation in their shape can be observed. ${ }^{1,6,8}$ On the other hand, pores with irregular shapes and various sizes and depths are normally the results of phase separation. ${ }^{16,20}$

Another approach to create surface porosity and texture is electrospinning of polymer solutions in association with a nonsolvent, resulting in nonsolvent-induced phase separation (NIPS). ${ }^{18,23}$ The nonsolvent can be incorporated as vapor; ${ }^{1,8,20}$ used as a coagulation bath wherein the collector is placed, ${ }^{22}$ or directly mixed with the polymer solution before electrospinning. $5,12,14,16,20,24$ The direct incorporation of nonsolvent into polymer solutions, as we do here, can increase the reproducibility because the phase separation within the system is defined by the initial ternary composition. ${ }^{16}$ The fibers produced via electrospinning of a polymer-solvent-nonsolvent system may exhibit not only a rough, porous surface ${ }^{12}$ but also a porous core, which can enhance the performance of the membrane as an absorbent material. ${ }^{5}$

The pore formation and crystallization mechanisms during electrospinning of polymer-solvent-nonsolvent systems are not fully explored, most likely due to the complexity of the process as there are many parameters involved. ${ }^{1,8,16}$ Furthermore, due to the rapid nature of electrospinning, the thermodynamic rules for such ternary systems can be overridden by kinetics. Also, electrospinning of ternary systems is challenging as their viscoelastic properties prior to electrospinning, and consequently their electrospinnability, change over time as a result of phase separation. ${ }^{5,16}$ Advancement in phase separation increases the viscosity of the system until gelation occurs as a result of crystallization or vitrification of the polymer-rich phase. ${ }^{12,19-21}$ The impact of aging time during phase separation on viscoelastic properties and electrospinnability of nonsolvent-containing systems, ${ }^{12}$ which is overlooked in other studies, is taken into account in this study.

In our previous study, we determined the electrospinnability window (i.e., shelf time) of poly(lactic acid) (PLA)-dichloromethane (DCM; solvent)-hexane (nonsolvent) ternary systems based on SEM observations of the membranes. ${ }^{12}$ During the electrospinnability window, the system properties are such that a stable, continuous jet is formed leading to long, continuous and uniform fibers. ${ }^{7,11,25}$ Here, we introduce a rheological approach to identify the initiation of the electrospinnability window (i.e., lower limit of the shelf time ${ }^{12}$ ) for the phase-separating ternary systems prior to electrospinning. In order to reveal the different morphology formation mechanisms during the electrospinning of PLA-DCM-hexane and PLADCM systems, the crystallinity and pore morphology of the fibers produced from these systems were examined and compared with each other and with those of the corresponding NIPS-derived PLA foams. ${ }^{18}$ A solvent exchange step was also performed on the electrospun fibers to evaluate their performance in a typical nonsolvent at room temperature.

\section{EXPERIMENTAL SECTION}

2.1. Materials. PLA $\left(M_{\mathrm{w}}=194000\right.$ and D-lactide content $\left.=1.6 \%\right)$ was purchased from NatureWorks LLC (Ingeo Biopolymer 4032D). The molecular weight of this PLA was determined by the supplier 
based on solution viscosity measurements. Dichloromethane (DCM; Fisher Chemical; Stabilized/Certified ACS, >95.5\%) and hexane (Fisher Chemicals, Certified ACS, $>95 \%$ ) were respectively used as solvent and nonsolvent for PLA. Methanol (Fisher Chemicals; Certified ACS, 99.9\%) was also used for the solvent exchange process as another nonsolvent for PLA. The chemical structures and the physical and chemical properties of these materials are presented in Table 1.

2.2. Solution Preparation. The crystallinity of the as-received PLA was decreased via a melting-quenching process in order to facilitate its dissolution in solvent DCM. ${ }^{12,17,18}$ The resulting amorphous ( $95 \%)$ PLA was dissolved in DCM at $30{ }^{\circ} \mathrm{C}$ while mechanically stirring until a homogeneous solution was obtained. The evaporated amount of DCM (weight basis) was then added to the solution at room temperature. Note that all solutions were tightly sealed during preparation and storage. Binary solutions with three PLA concentrations of 9.1, 10.6, and 12.1 wt \% were prepared via this process (Table 2).

\section{Table 2. Binary and Ternary Systems Used in This Study}

\begin{tabular}{cccc}
$\begin{array}{c}\text { overall PLA concn of the } \\
\text { system (wt \%) }\end{array}$ & $\begin{array}{c}\text { system } \\
\text { type }\end{array}$ & $\begin{array}{c}\text { PLA in DCM } \\
(\text { wt } \%)\end{array}$ & $\begin{array}{c}\text { hexane/DCM } \\
(\mathrm{v} / \mathrm{v})\end{array}$ \\
9.1 & $\begin{array}{c}\text { binary } \\
\text { ternary }\end{array}$ & 9.1 & 0 \\
& binary & 13 & 1 \\
10.6 & ternary & 15 & 0 \\
& binary & 12.1 & 1 \\
12.1 & ternary & 17 & 0 \\
& & & \\
\hline
\end{tabular}

In order to prepare the ternary systems with the same PLA concentrations of 9.1, 10.6, and 12.1 wt \%, first PLA-DCM solutions with appropriate concentrations $(13,15$, and 17 wt $\%$, respectively) (Table 2) were prepared via the process explained above. The nonsolvent hexane was then gradually added to the solutions while stirring under ambient conditions to reach hexane/DCM volume ratio of $1(\mathrm{v} / \mathrm{v})$ (Table 2). These ternary systems are located in the liquidliquid phase separated region of the PLA-DCM-hexane phase diagram (Figure S1 in the Supporting Information). ${ }^{12,17,18}$

2.3. Evaporation Experiments. The rate of solvent evaporation during electrospinning and thereafter has a significant impact on pore formation and crystallization. Thus, a series of evaporation tests were performed on ternary and binary systems in order to compare their evaporation rates under the same ambient conditions as those for the electrospinning processes: $23{ }^{\circ} \mathrm{C}$ and $\sim 50 \%$ relative humidity. Samples of each system with the same volume were prepared in $20 \mathrm{~mL}$ vials (bottom diameter of $26.8 \mathrm{~mm}$ ), placed on a shelf, and exposed to air. The weight of the vials was recorded every 30 min over a $13 \mathrm{~h}$ time period. The change in mass of each system relative to the initial mass was then plotted as a function of time.

2.4. Rheological Experiments. Rheological studies provide information on viscoelastic properties of systems which we use to explain their electrospinnability. The rheological experiments were performed using an Anton Paar (MCR 502) rheometer and a Couette geometry. A solvent trap (Anton Paar; ST-CYL-C/Q1) which can be assembled on the Couette geometry was also used to minimize the evaporation of DCM and hexane in our system. The results of a time sweep on the binary solution with 10.6 wt \% PLA revealed that in spite of using the solvent trap, there was a noticeable evaporation starting after $3 \mathrm{~h}$, reflected as a significant increase in viscosity from 1.5 to $10^{4}$ $\mathrm{Pa} \cdot \mathrm{s}$ over $\sim 1.5 \mathrm{~h}$. Under these conditions, a tall skin was observed on the bob as a result of rod climbing (Figure S2). The rod climbing can be partially attributed to the capillary forces which are also promoted by the gradual evaporation of solvent from the system. Thus, additional measures were taken into account including further sealing of the solvent trap using carbon tape and parafilm as well as low viscosity silicone oil $(\sim 1.5 \mathrm{~mL})$. These measures are explained in more detail in the Supporting Information (Figure S2). After applying all of these measures, rod climbing was minimized and a much smaller increase in viscosity (starting after $13 \mathrm{~h}$ from $\sim 1$ to $40 \mathrm{~Pa} \cdot \mathrm{s}$ over $10 \mathrm{~h}$ ) was observed for the binary system (10.6 wt \%) (Figure S3). Thus, all the rheological experiments were carried out using this setup as explained below.

First, a strain sweep between $0.1 \%$ and $10 \%$ confirmed that at less than $1 \%$ strain the systems remain within their linear viscoelastic region. The rheological behavior of all ternary systems and one of the binary systems (10.6 wt \%) was evaluated via time sweeps at $1 \%$ strain under ambient conditions by loading $4.6 \mathrm{~mL}$ of solution in a Couette configuration. The time sweep tests were repeated three times for each concentration system, and the average values were obtained. Then, for the ternary systems, the data were normalized to the average of complex viscosity values prior to the induction time of crystallization. Note that the time sweep curves are shifted by $75 \mathrm{~min}$, which is the time between the sample preparation and loading the rheometer.

2.5. Electrospinning. The binary and ternary systems (Table 2 ) were electrospun under $25 \mathrm{kV}$ applied by a high-voltage power supply (Spellman, model SL150). Each ternary system was electrospun at

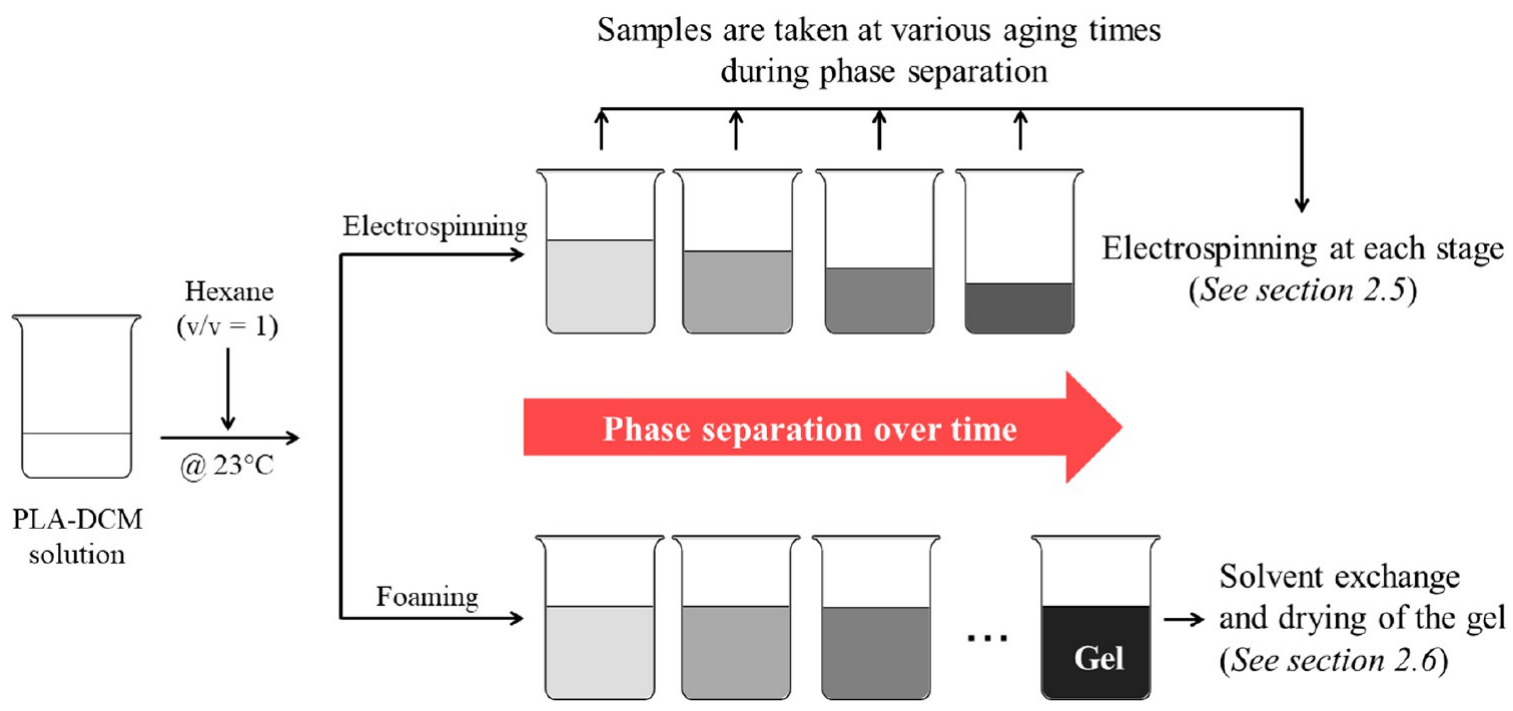

Figure 1. Electrospinning of membranes (top route) versus foaming (bottom route) from phase-separated ternary systems. The reason that the height of the liquid in the top route is shown to be decreasing is that samples are taken from the system at various times for electrospinning. Note that the containers must be tightly sealed at all times as explained in section 2.2. 


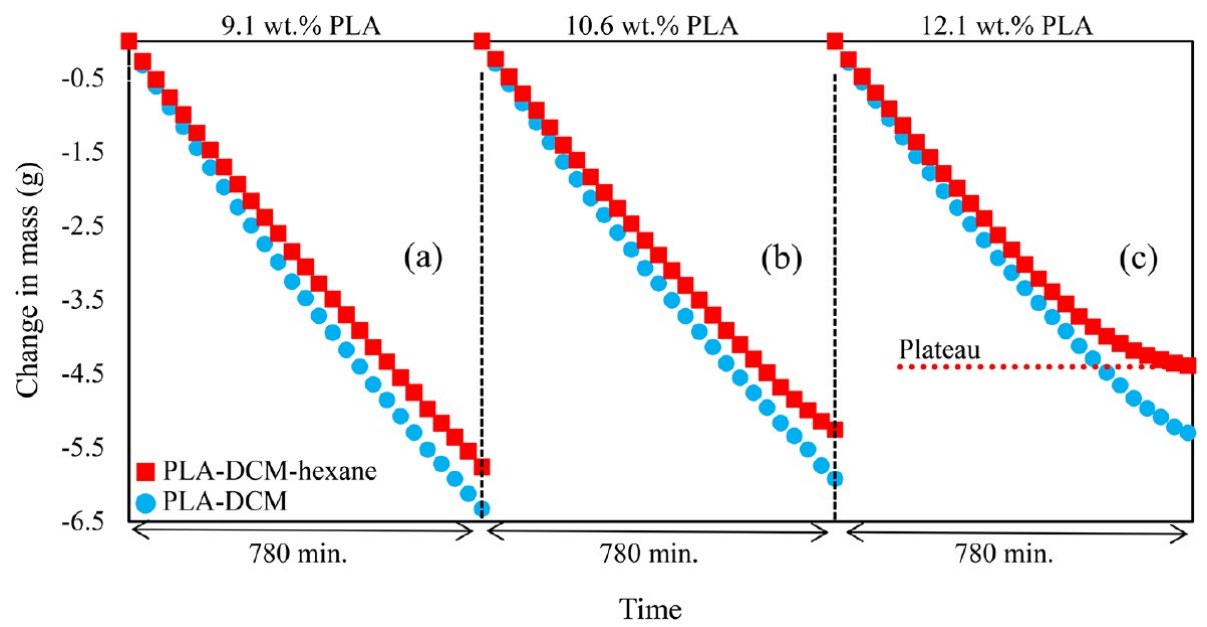

Figure 2. Results of evaporation tests (change in mass relative to initial mass) for ternary and binary systems with various PLA concentrations: 9.1 wt $\%$ (a), 10.6 wt \% (b), and 12.1 wt \% (c) (Table 2). The time interval between measurements is 30 min as described in section 2.3 .

various aging times (during its phase separation prior to gelation (Figure 1). ${ }^{12}$ The ambient and processing parameters were kept constant for all electrospinning experiments. The temperature and environmental humidity were $23{ }^{\circ} \mathrm{C}$ and $\sim 50 \%$, respectively. The process was performed at a flow rate of $5 \mathrm{~mL} / \mathrm{h}$ adjusted by a digital syringe pump (Harvard Apparatus, model 703307 R) using a stainless steel, 21 gauge needle. The distance between the tip of the nozzle and the collector (working distance) was fixed at $10 \mathrm{~cm} .{ }^{12}$

The electrospinning experiments must be performed quickly after loading the syringe in order to minimize evaporation. A piece of cotton containing a few droplets of hexane and DCM (v/v = 1) was hung inside the vials to compensate for the evaporation caused by opening the containers to take portions of the ternary system for each electrospinning experiment. The high volatility of DCM and hexane $^{12,17}$ also resulted in unavoidable formation of skin/gel at the tip of the syringe which required frequent removal in order to maintain a stable jet flow and avoid the formation of more than one jet string with smaller diameters (i.e., jet branching). ${ }^{6}$

The collector was covered with new aluminum foil before each experiment, and the aluminum foil with the deposited membrane was collected for further characterization or solvent exchange. The membranes produced from the binary systems and some of the membranes produced from the ternary systems were immediately cut in half after fabrication. The first half was allowed to dry in air, and the other half was immersed in methanol for $4 \mathrm{~h}$ for solvent exchange followed by drying in air. The same solvent exchange process was also performed on the membranes electrospun from binary systems after 2 weeks aging under ambient conditions.

2.6. Foaming. PLA foams were also produced from the three ternary systems (Table 2) to compare their properties with those of the membranes electrospun from the same systems. In order to produce PLA foams, the ternary systems (Table 2), which were prepared as explained in section 2.2 , were allowed to completely phase separate until they gel (Figure 1). The gelation time (measured in a sealed container ${ }^{18}$ ) of the ternary systems with PLA concentrations of 9.1, 10.6, and 12.1 wt $\%$ is $3 \pm 0.5$ days, $24 \pm 3 \mathrm{~h}$, and $8 \pm 2 \mathrm{~h}$, respectively. ${ }^{18}$ The resulting gels were aged for $\sim 24 \mathrm{~h}$ and then carefully demolded and cut. The gel cubes were immersed in methanol for $30 \mathrm{~h}$ solvent exchange followed by air drying. This NIPS foaming process is described in more detail in our previous work. ${ }^{18}$

2.7. Characterization. The electrospun membranes and PLA foams (before and after solvent exchange) were characterized by differential scanning calorimetry (DSC; TA Instruments, Q200) and scanning electron microscopy (SEM; HITACHI, S-3400N). After an overnight degassing step, the specific surface area of the membranes electrospun from binary and ternary systems with PLA concentration of $10.6 \mathrm{wt} \%$, with and without solvent exchange, was measured by the
Brunauer-Emmett-Teller (BET) $\mathrm{N}_{2}$ adsorption-desorption test (Tristar $3000 \mathrm{~V} 6.07$ ) at $77.3 \mathrm{~K}$.

The DSC experiments were performed in sealed aluminum crucibles under a nitrogen atmosphere as heating scans from 25 to $250{ }^{\circ} \mathrm{C}$ at a heating rate of $5{ }^{\circ} \mathrm{C} / \mathrm{min}$. However, only the parts of the curves containing useful information $\left(40-180^{\circ} \mathrm{C}\right)$ are presented here. All DSC data are the average of two measurements for each sample. The surface texture ${ }^{12}$ and the core morphology of the electrospun fibers and the pore structure of the fracture surface of the foams ${ }^{18}$ were examined by SEM under high vacuum. Note that the membranes produced from ternary systems at various aging times were examined by SEM in our previous study. ${ }^{12}$ In order to observe the core structure of the fibers, the membranes were cut using a sharp blade while soaking in liquid nitrogen. Prior to SEM examination, all the specimens were mounted on carbon tapes placed on an aluminum stand and then coated with gold for $120 \mathrm{~s}$ using a rotary-pumped sputter coater (Quorum, Q150R ES).

All membranes were also quantitatively examined in terms of the size of their fibers by applying ImageJ software to the SEM images, before and after solvent exchange. For each membrane, the diameters of at least 50 randomly selected fibers were measured and the average values are reported.

\section{RESULTS AND DISCUSSION}

3.1. Evaporation Experiments. The evaporation of DCM and hexane can have a significant influence not only on the rheological experiments but also on the morphology formation during the electrospinning process. Figure 2 presents the results of the evaporation tests for binary and ternary systems as change in mass as a function of time. In general, the evaporation rate depends on the combination of the degree of volatility of the solvents and the polymer-solvent interaction parameters which are further explained in section 3.3. This process involves the diffusion of the solvent to the surface where it then evaporates. According to Figure 2, for all concentrations, the binary solutions evaporate faster than the ternary systems.

In binary solutions, DCM diffuses to the surface and evaporates as a result of its high vapor pressure (Table 1 ). In ternary systems, the diffusion of the polymer-lean phase (hexane + DCM) to the surface is expected to be faster because of the branchlike channels formed as a result of phase separation. In spite of this faster diffusion, the overall evaporation rate for the ternary systems is lower (Figure 2) because the vapor pressure of the polymer-lean phase (hexane 


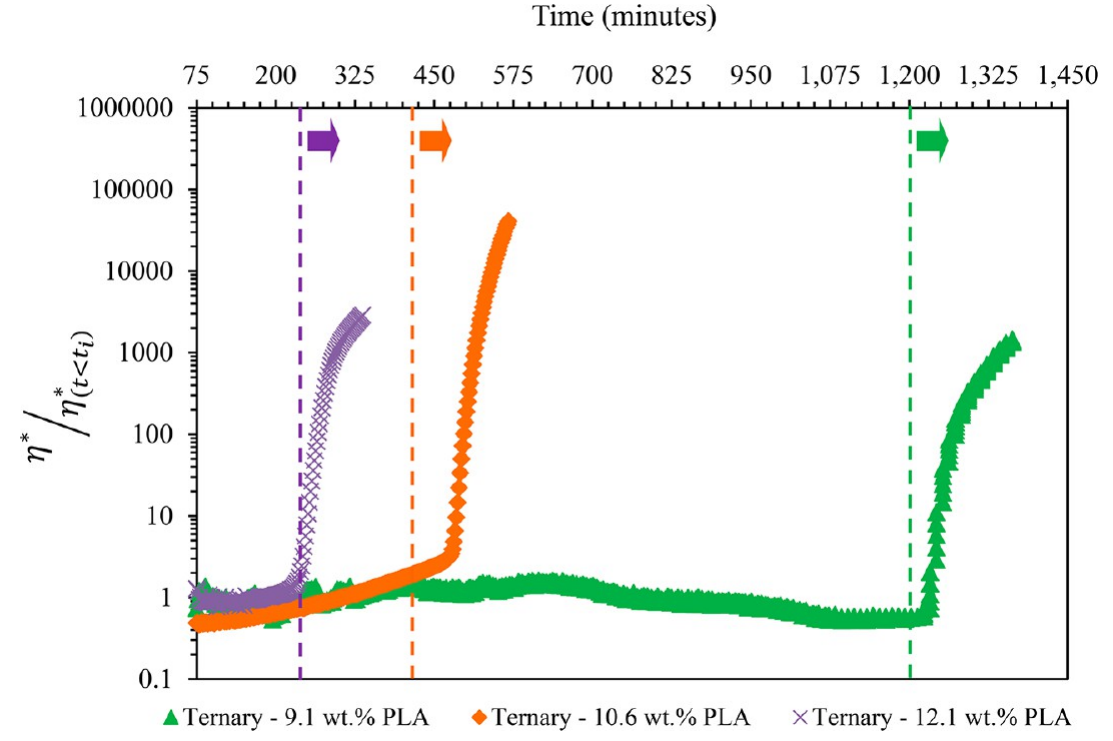

Figure 3. Complex viscosity as a function of time for the ternary systems. The beginning of the electrospinnability window for the ternary systems ${ }^{12}$ is indicated with the dashed lines which are close to the induction time of crystallization (rapid increase in viscosity) of the corresponding system. Each curve is normalized to the average of complex viscosity values prior to the induction time of crystallization $\left(t_{\mathrm{i}}\right)$.

+ DCM) is lower than that of pure DCM in the binary systems (Table 1). ${ }^{9}$ In other words, the polymer-lean phase in ternary systems may reach the surface faster than that of DCM in binary systems, but it takes longer for it to evaporate. Also, as the PLA concentration of systems increases (a to c in Figure 2), the evaporation rate decreases such that for the ternary system with PLA concentration of $12.1 \mathrm{wt} \%$ the system almost reaches a plateau within the test period as it gels.

Although these results (Figure 2) provide an insight about the evaporation rates of the binary and ternary systems, it must be noted that evaporation during electrospinning is much faster than in these tests due to the rapid increase in specific surface area of the system within milliseconds. The results of evaporation experiments are used in the following section to explain the rheological behavior of the systems as well as the pore formation and crystallization mechanisms of electrospun membranes.

3.2. Rheology. For solution electrospinning, there is a critical concentration which results in sufficient chain entanglement and therefore a high enough viscosity for electrospinnability. ${ }^{5,7,11}$ As explained in the Introduction, producing uniform, continuous fibers via electrospinning of polymer-solvent-nonsolvent systems can be challenging due to the changing viscoelastic properties of the phase-separating systems prior to electrospinning. ${ }^{5,7,16}$ Rheological studies provide information on viscoelastic properties of systems and explain the transition from electrospraying to successful electrospinning for various solutions. ${ }^{7,25}$

The complex viscosity of the ternary systems as a function of time is presented in Figure 3. The induction time of crystallization which corresponds to the drastic increase in viscosity of the ternary systems (Figure 3 ) is 1220, 470, and 225 min for systems with PLA concentrations of 9.1, 10.6, and 12.1 wt \%, respectively. For these systems, chains in the polymerrich phase start to order at the induction time of crystallization when it is concentrated enough. ${ }^{18,19,21}$ Further solvent diffusion accelerates the ordering. These semiordered regions act as nuclei for further crystallization later on in the process which is explained further in section 3.4.
The complex viscosity of the PLA-DCM binary solution with $10.6 \mathrm{wt} \%$ PLA as a function of time is presented in Figure S3. No sudden increase in viscosity can be observed for this binary system, and the gradual increase in its viscosity is solely due to evaporation over the long-term experiment. Thus, evaporation still plays a role in our rheological experiments (Figure 3), although its impact in increasing the viscosity of the ternary systems is much less significant than that of the crystallization within their polymer-rich phase. Also, note that less evaporation is expected for the ternary systems according to the evaporation studies (Figure 2).

The beginning of the electrospinnability window (i.e., shelf time $^{12}$ ) for the ternary systems, as determined in our previous study $^{12}$ based on SEM images of the membranes, is indicated in Figure 3. The electrospinnability window starts at 1200, 420, and $240 \mathrm{~min}$ and ends at 1620, 1320, and $360 \mathrm{~min}$ after mixing hexane with PLA-DCM solutions for systems with 9.1, 10.6, and $12.1 \mathrm{wt} \%$ PLA, respectively. ${ }^{12}$ As mentioned previously, the electrospinnability window starts when the viscoelastic properties of the phase separating system reach optimum conditions to form a stable, continuous jet flow resulting in long, uniform fibers. ${ }^{12}$ The electrospinnability window ends as large polymer-lean regions are formed within the phaseseparated system resulting in an overall lack of chain entanglement throughout the system. ${ }^{12,21}$ These larger polymer-lean domains rapidly evaporate during the jet flying, and there remains behind a heterogeneous system leading to the rupture of the jet flow. ${ }^{16}$

According to Figure 3, the electrospinnability window of the ternary systems studied here starts when the systems are close to their induction time of crystallization. The largest difference between the beginning of the electrospinnability windows and their corresponding induction times of crystallization is observed for the ternary system with overall PLA concentration of 10.6 wt \% (Figure 3). For this system, the electrospinnability starts at $\sim 420 \mathrm{~min}$, which is about $10 \%$ apart from the induction time of crystallization for this system (470 $\mathrm{min}$ ). Thus, the electrospinnability of the phase-separating ternary systems may be related to the initiation of chain ordering and 


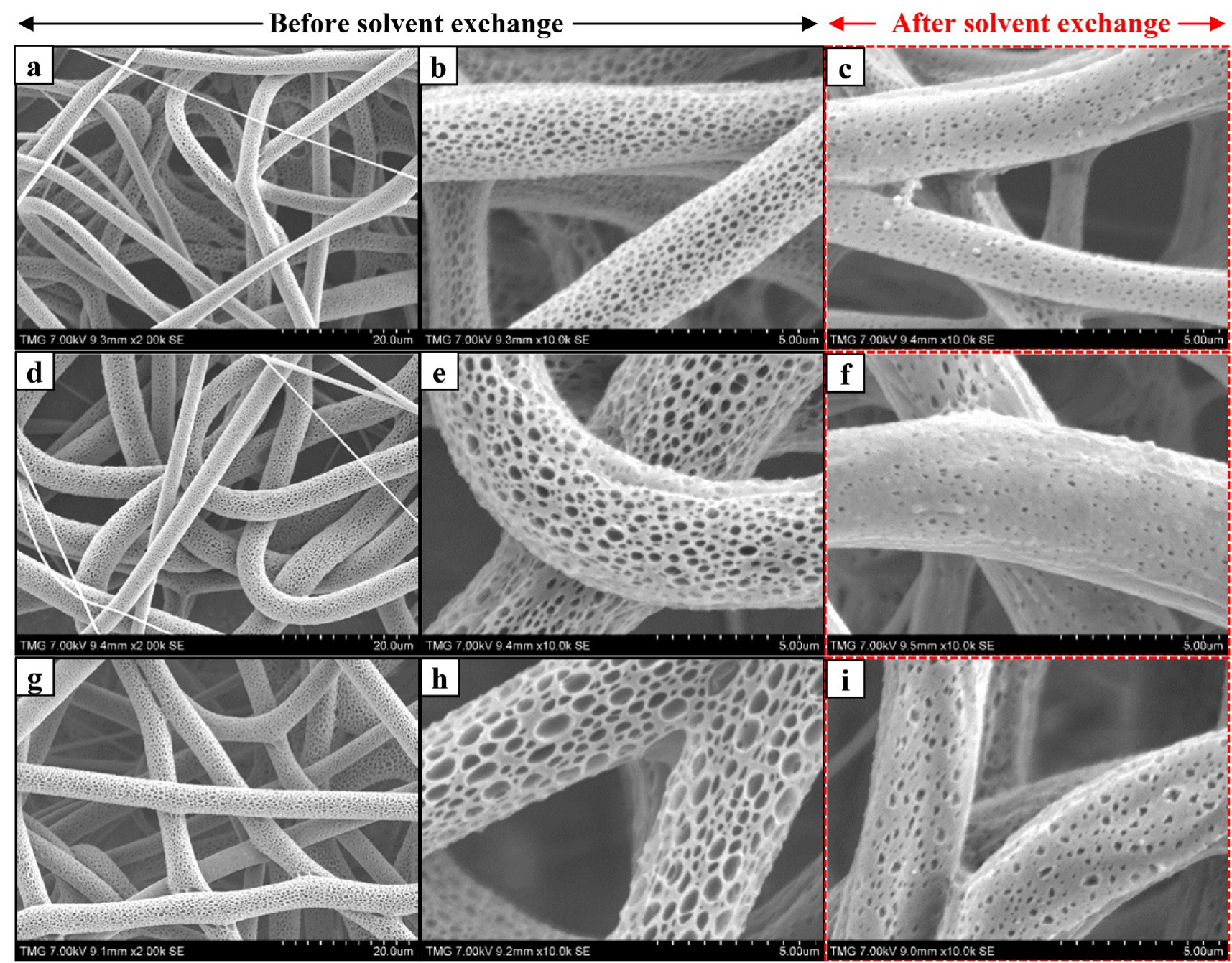

Figure 4. SEM images of membranes electrospun from binary systems with PLA concentrations of 9.1 wt \% (a-c), 10.6 wt \% (d-f), and 12.1 wt \% $(g-i)$. The middle column (b, e, and h) contains enlargements of the left column ( $a, d$, and g). The right column (c, f, and i) shows the morphology of the respective fibers in the middle column after solvent exchange (performed immediately after the deposition).

formation of incipient crystals within the polymer-rich phase. Note that by changing the processing and ambient conditions, the electrospinnability of the systems also changes. ${ }^{1,3,12} \mathrm{We}$ further discuss the electrospinnability of our ternary systems based on the chain entanglement concept as well as the SEM images of their electrospun membranes in the next section.

3.3. Morphology. In this section, first, we discuss the morphology of and the pore formation mechanisms for the fibers electrospun from binary systems and then we discuss and compare these matters for the ternary systems.

3.3.1. Membranes Electrospun from Binary Systems. The morphology of the fibers electrospun from the binary PLADCM solutions is presented in Figure 4. The surface of these fibers is packed with pores of various sizes, especially for those fibers produced from solutions with higher concentrations (e.g., $12.1 \mathrm{wt} \%$, Figure $4 \mathrm{~h})$. Note that this highly porous structure is formed under a high voltage $(25 \mathrm{kV})$, even though high voltages are known to decrease the surface porosity of electrospun fibers. ${ }^{9,14}$ Bognitzki et al. ${ }^{6}$ electrospun PLA fibers from a 5 wt $\%$ DCM solution with similar surface porosity to those in the left and middle columns of Figure 4. However, the pore size distribution for their fibers is narrower compared to that of our fibers. The wider surface pore size distribution on our fibers indicates that, in addition to breath figures, other mechanisms such as TIPS are playing a role. As explained in the Introduction, the surface pores caused by the breath figures mechanism are expected to be similar in shape and size, whereas the pores formed as a result of phase separation (TIPS) are normally more irregular in shape and size. The presence of such pores with various sizes on the surface of our fibers (i.e., the wider surface pore size distribution) indicates that the pores are formed not only by the breath figures mechanism but also by TIPS. ${ }^{1,16,20}$ Water is partially miscible in $\mathrm{DCM}^{16}$ and thus can form pores via NIPS as well. ${ }^{9}$ The high volatility of DCM (Table 1 ) is essential to activate both breath figures and TIPS mechanisms. ${ }^{1,6}$

The surface porosity of the fibers electrospun from binary solutions is decreased when solvent exchange is performed immediately after membrane deposition (Figure 4c,f,i). As a result, for example, the specific surface area of the membrane produced from the binary system with PLA concentration of 10.6 wt \% (Figure $4 \mathrm{~d}, \mathrm{e}$ ) is also decreased from 0.68 to $0.18 \mathrm{~m}^{2} /$ $\mathrm{g}$ after the solvent exchange. In spite of the high volatility of DCM and the high surface area of the fibers, the remaining DCM, which is mostly within the core of the fibers, is large enough to cause morphological change during solvent exchange at room temperature. DCM is a very good solvent for PLA, as indicated by the solubility parameter, making the removal of 


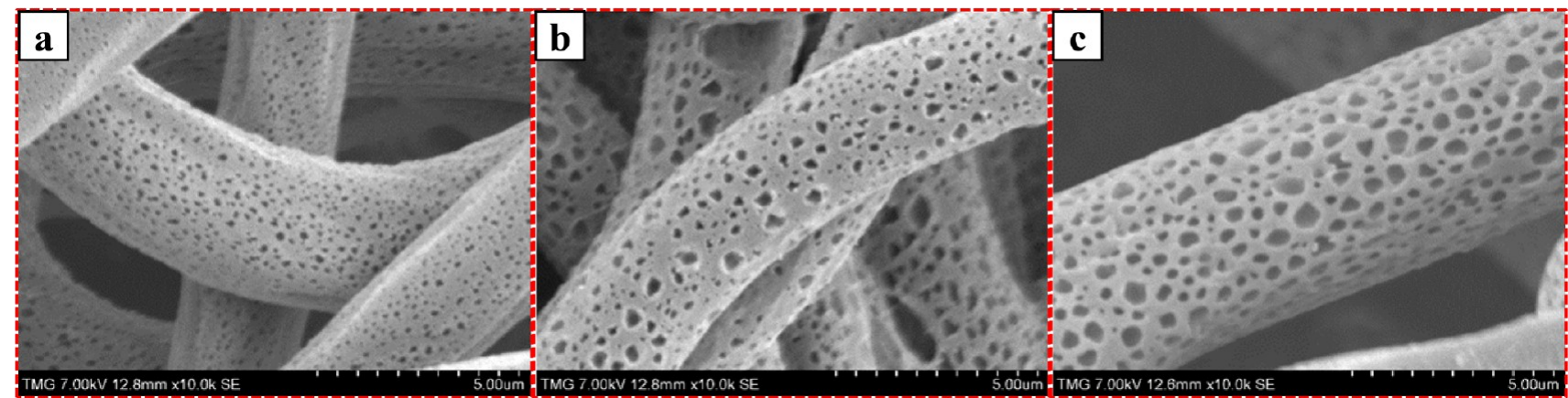

Figure 5. SEM images of the membranes electrospun from binary systems with PLA concentrations of 9.1 wt \% (a), 10.6 wt \% (b), and 12.1 wt \% (c) which were aged for 2 weeks before solvent exchange as described in section 2.5 . Note that images a, b, and c respectively correspond to images $\mathrm{a} / \mathrm{b} / \mathrm{c}, \mathrm{d} / \mathrm{e} / \mathrm{f}$, and $\mathrm{g} / \mathrm{h} / \mathrm{i}$ of Figure 4 .

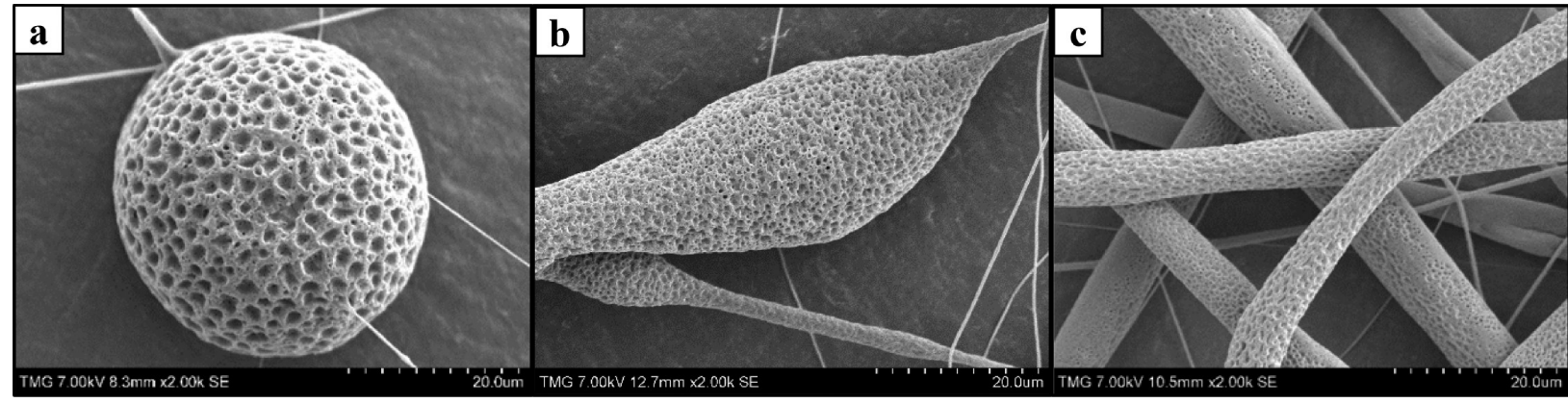

Figure 6. SEM images of the electrospun materials from the ternary system with PLA concentration of 9.1 wt \% at various aging times during phase

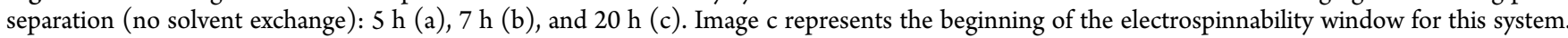
Note that further morphological examinations on the membranes electrospun from ternary systems at various aging times are presented in ref 12 . SEM images of higher magnifications from image (a) better showing its pore morphology are presented in the Supporting Information (Figure S4).

residual DCM more difficult. During electrospinning, the rapid DCM evaporation occurs on the surface of the fibers while the residual solvent in the core gradually diffuses to the surface to evaporate. This rapid surface evaporation leads to a high concentration of polymer chains on the surface which eventually forms the skin. ${ }^{1,16,20}$ This skin hinders the diffusion and evaporation of the residual DCM. The DCM-methanol mass transfer during solvent exchange facilitates the diffusion of the residual $\mathrm{DCM}^{22}$ which lowers the glass transition temperature $\left(T_{g}\right)$ of the PLA on the surface, in particular the amorphous chains of the skin. This process allows the pores to reshape and shrink. The hydrogen bond network between the loosely attached, amorphous chains of the skin may also favor pore shrinkage. ${ }^{9}$ Similar surface morphologies to those of the fibers after solvent exchange (Figure $4 \mathrm{c}, \mathrm{f}, \mathrm{i}$ ) are reported for the poly(acrylonitrile) fibers electrospun directly into a nonsolvent bath. $^{22}$

Figure 5 presents the SEM images of the fibers electrospun from binary systems which were aged for 2 weeks before the solvent exchange step. The model explained above is consistent with these SEM images (Figure 5), revealing that the surface porosity of the membranes which were aged for 2 weeks before solvent exchange is less affected by this process. During this aging period, residual DCM evaporates slowly from the core of the fibers through the skin without disturbing the pore structure.

From the above discussion, it can be concluded that even for systems containing volatile solvents such as DCM, the surface porosity of the fibers may not be fully stable after deposition for subsequent applications. These observations also highlight the importance of the polymer-solvent interaction parameter which determines the degree of solubility of a polymer in a solvent. $^{18,20,26}$ The Flory-Huggins interaction parameter $(\chi)$ between the two species can be calculated from eq $1 .{ }^{17,20,26,27}$

$$
\chi_{i j}=\frac{V_{\mathrm{r}}}{R T}\left(\delta_{i}-\delta_{j}\right)^{2}
$$

where $V_{\mathrm{r}}$ is the reference molar volume and $\delta$ is the solubility parameter. ${ }^{17,25,27}$ The solubility parameters for PLA, DCM, hexane, and methanol at $25^{\circ} \mathrm{C}$ are $10.10,10.54,7.24,{ }^{17}$ and $14.5^{26}\left(\mathrm{cal} / \mathrm{cm}^{3}\right)^{1 / 2}$, respectively. As expected, DCM is a good solvent and hexane is a nonsolvent for PLA, since $\left|\delta_{\mathrm{PLA}}-\delta_{\mathrm{DCM}}\right|$ $=0.44\left(\mathrm{cal} / \mathrm{cm}^{3}\right)^{1 / 2}$ and $\left|\delta_{\text {PLA }}-\delta_{\text {hexane }}\right|=2.86(\mathrm{cal} /$ $\left.\mathrm{cm}^{3}\right)^{1 / 2} \cdot 17,20,26$ Also, methanol, which is used in the solvent exchange process, is an excellent nonsolvent for PLA as $\mid \delta_{\mathrm{PLA}}-$ $\delta_{\text {hexane }}|<| \delta_{\text {PLA }}-\delta_{\text {methanol }} \mid=4.4\left(\mathrm{cal} / \mathrm{cm}^{3}\right)^{1 / 2}$.

The polymer-solvent interaction parameter depends on temperature and the chemical composition of the components. ${ }^{17,20,26}$ For a solution of a polymer in a good solvent (small $\chi$ ), the polymer chains swell to enhance the intermolecular interactions unlike a poor solvent which favors the contraction of the chains. ${ }^{11}$ For a solution with small $\chi$ such as our PLA-DCM, solvent evaporation can be hindered due to the high level of intermolecular interactions between the polymer and solvent, especially at higher polymer concentrations (Figure 2). Additionally, PLA and DCM form intermolecular hydrogen bonds (molecular structures in Table 1), making the removal of remaining DCM even more difficult.

3.3.2. Membranes Electrospun from Ternary Systems. Figure 6 presents the SEM images of the materials electrospun from ternary systems at various aging times during phase 

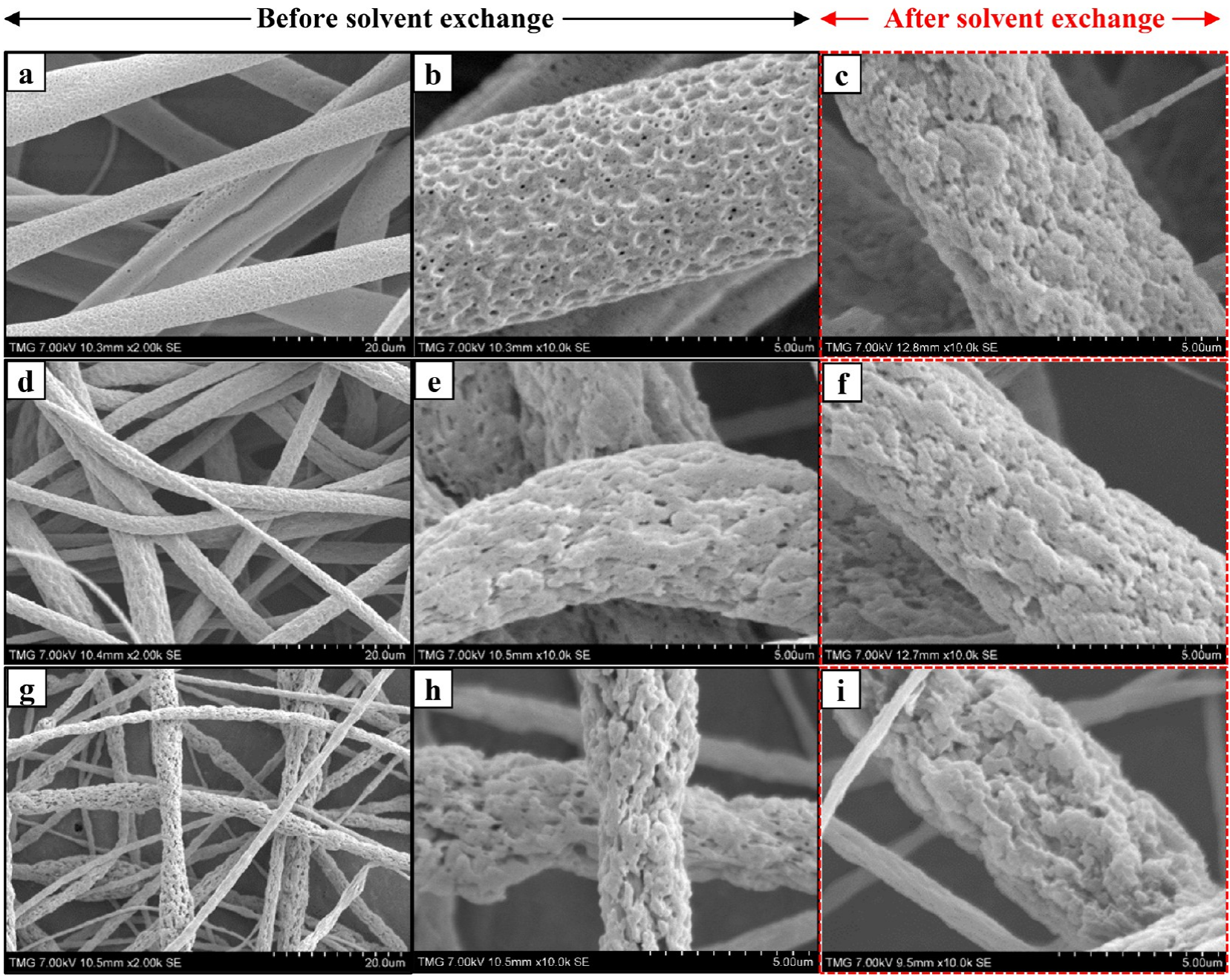

Figure 7. SEM images of membranes electrospun from ternary systems with PLA concentrations of $9.1 \mathrm{wt} \%(\mathrm{a}-\mathrm{c}), 10.6 \mathrm{wt} \%$ (d-f), and $12.1 \mathrm{wt} \%$ $(g-i)$. The middle column $(b, e$, and $h)$ contains enlargements of the left column ( $a, d$, and $g)$. The right column (c, f, and $i)$ shows the morphology of the respective fibers in the middle column after solvent exchange (performed immediately after the deposition). Each ternary system is electrospun at the late stages of its electrospinnability window.

separation. These SEM images show the significant effect of aging during phase separation on the morphology of the electrospun fibers. ${ }^{12}$ Electrospinning of the ternary systems at early stages of phase separation, before the electrospinnability window starts, may result in the formation of microbeads and bead-on-string morphology (Figure 6a,b).

The striking difference between the microbeads morphology (Figure 6a) and the perfect fibers electrospun from the corresponding binary system (9.1 wt \%) (Figure 4a) reveals an important fact: the incorporation of the nonsolvent may increase the surface tension of the system compared to that of the binary solution, ${ }^{12,16,24}$ favoring the formation of the microbeads at this stage. More importantly, the lack of chain entanglement in the polymer-lean phase reduces the effective resistance to flow, leading to the formation of microbeads and bead-on-string morphology. $5,7,11,12,29$ The chain entanglement in a polymer solution increases by increasing the polymer concentration and/or the solvent quality. ${ }^{7}$ Although the polymer concentration in the polymer-rich phase in the ternary system of Figure 6a may be high enough to allow for chain entanglement, there is certainly no entanglements in polymerlean domains wherein no chain overlapping exists. ${ }^{7}$ On the other hand, there is a uniform distribution of chain entanglement throughout the corresponding binary system resulting in perfect fibers (Figure 4a).

The pore formation process during electrospinning of the ternary systems is complex as hexane-induced phase separation is also introduced to the system. The morphology of the fibers electrospun from the ternary systems is presented in Figure 7. The surface of these fibers has a very rough texture, in particular for those produced from higher concentration systems (Figure $7 \mathrm{e}, \mathrm{h})$. For the low concentration ternary system (9.1 wt \%), breath figures, TIPS, and humidity induced phase separation still play an important role in the pore formation similar to that of the binary systems. Note that the incorporation of hexane, which is highly hydrophobic, also diminishes the humidity incorporation into the system. For the systems with higher PLA concentrations (10.6 and $12.1 \mathrm{wt} \%)$, the hexane-induced phase separation becomes the predominant factor controlling the formation of the surface roughness (Figure 7e,h). Thus, there is a critical concentration for the ternary systems at which surface porosity of the fibers coarsens to surface roughness.

Qi et al. ${ }^{14}$ produced highly porous PLA fibers with a morphology similar to that of our fibers (Figure 7e,h). They used systems containing PLA, dichloromethane (solvent), and butanol (nonsolvent) with 8 wt \% PLA and various 


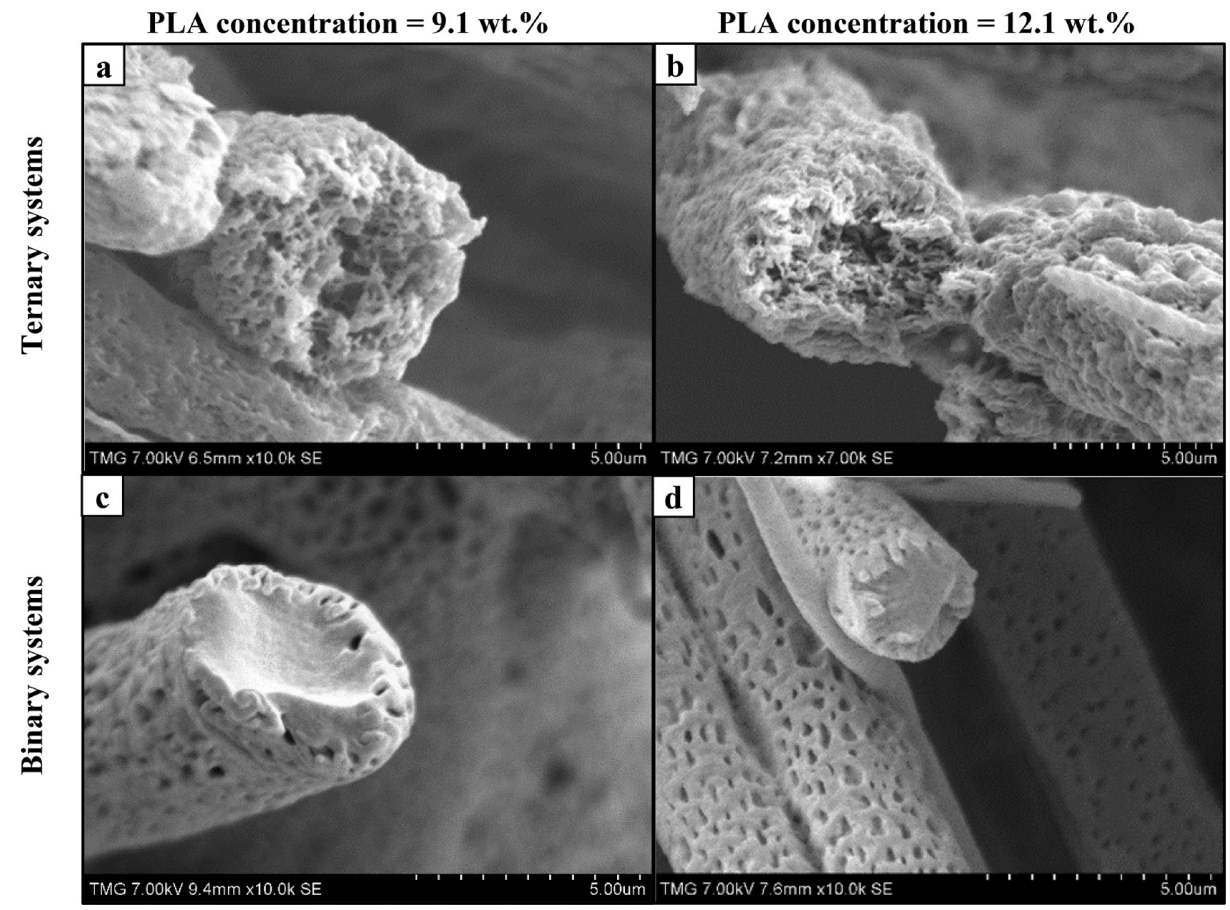

Figure 8. SEM images of the core of the fibers electrospun from ternary (9.1 wt \% (a) and 12.1 wt \% (b) PLA concentration) and binary (9.1 wt \% (c) and $12.1 \mathrm{wt} \%$ (d) PLA concentration) systems (before solvent exchange). The fibers of images a and b are electrospun at the late stages of their electrospinnability window and correspond to the images of Figures $7 \mathrm{~b}$ and $7 \mathrm{~h}$, respectively. Note that soaking in liquid nitrogen during the cutting process (described in section 2.7) may have slightly affected the morphology of the fibers.

nonsolvent/solvent ratios. They showed that if the solvent and nonsolvent have similar volatility, the porosity and roughness of the fibers are drastically diminished. Thus, the different volatility of DCM and hexane (Table 1) may be essential in the formation of surface roughness in our systems. ${ }^{14,16}$

Unlike with the binary systems, solvent exchange does not have a significant effect on the surface morphology of the fibers (Figure $7 \mathrm{e}, \mathrm{f}, \mathrm{h}, \mathrm{i}$ ), except for the membrane produced from the low concentration (9.1 wt \%) ternary system (Figure $7 b, c)$. The porous surface of the fibers electrospun from ternary system of $9.1 \mathrm{wt} \%$ (Figure $7 \mathrm{~b}$ ) converts to surface roughness after solvent exchange (Figure 7c) as the hexane-induced phase separation within the fibers is continued by the methanolinduced phase separation during solvent exchange. ${ }^{22}$ In comparison with the binary systems, there may be less residual DCM in the core of the electrospun fibers produced from the ternary systems due to their branch-like, channeled phaseseparated structures (Figure 8a,b) as well as more open pores on their surface (Figure $7 \mathrm{e}, \mathrm{h}$ ) facilitating the diffusion of the solvents from the core to the surface.

The specific surface area of the membrane produced from the ternary system with PLA concentration of $10.6 \mathrm{wt} \%$ (Figure $7 \mathrm{~d}, \mathrm{e}$ ) is $3.82 \mathrm{~m}^{2} / \mathrm{g}$, which is more than 5 times higher than that of the membrane produced from the corresponding binary system. This indicates that the surface roughness of the membranes of ternary systems may provide a larger surface area than that of the surface porosity of the membranes of the binary systems. Even though no obvious morphological change is observed in the SEM images of the fibers of ternary systems (10.6 and $12.1 \mathrm{wt} \%$ ) after solvent exchange, there is a decrease in the specific surface of this membrane from 3.82 to $1.89 \mathrm{~m}^{2} / \mathrm{g}$ as a result of solvent exchange.

From Figures 4 and 7, we can conclude that the PLA fibers electrospun from high concentration ternary systems may be more stable in terms of surface morphology in comparison with that of the binary systems. Also, the surface of the fibers produced from binary systems is smoother (Figure $4 \mathrm{~b}, \mathrm{e}, \mathrm{h}$ ) compared to that of the ternary systems (Figure $7 \mathrm{~b}, \mathrm{e}, \mathrm{h}$ ), in particular at higher PLA concentrations.

The fibers electrospun from ternary and binary systems are also drastically different in terms of their core morphology respectively having a porous and dense core structures (Figure 8). The pore formation mechanisms active in binary systems only contribute to the formation of surface porosity and texture and have no effect on the core of the fibers (Figure $8 c, d$ ). However, the hexane-induced phase separation in ternary systems creates fibers with a highly interconnected porous core (Figure $8 \mathrm{a}, \mathrm{b}$ ) in addition to a rough surface texture. ${ }^{20,24}$

Similar to the binary systems, during the electrospinning of the ternary systems, the rapid evaporation first occurs on the surface of the fibers. DCM is more volatile than hexane (Table $1)$; thus, the surface composition of the jet is more rapidly brought to the hexane-rich side of the phase diagram (Figure S1), crossing into the unstable conditions where spinodal demixing is favored. $1,5,14,16,17,20,21$ Meanwhile, the core of the fibers is still in the metastable region where the system phase separates via nucleation and growth. As hexane and DCM gradually diffuse out from the core, the surface of the fibers may be brought to the metastable region. ${ }^{1,6,20,26}$ Thus, the pore formation in the ternary system, which is mostly the result of hexane-induced phase separation, is driven by a combination of spinodal and nucleation and growth demixing.

3.3.3. Average Diameter of the Fibers (Image Analysis). In addition to the qualitative examination and comparison in the previous sections, all membranes are quantitatively analyzed in terms of the average size (diameter) of their fibers in this section. The results of the image analysis on SEM images of the membranes (Figure 9) further elaborate the effect of phase 


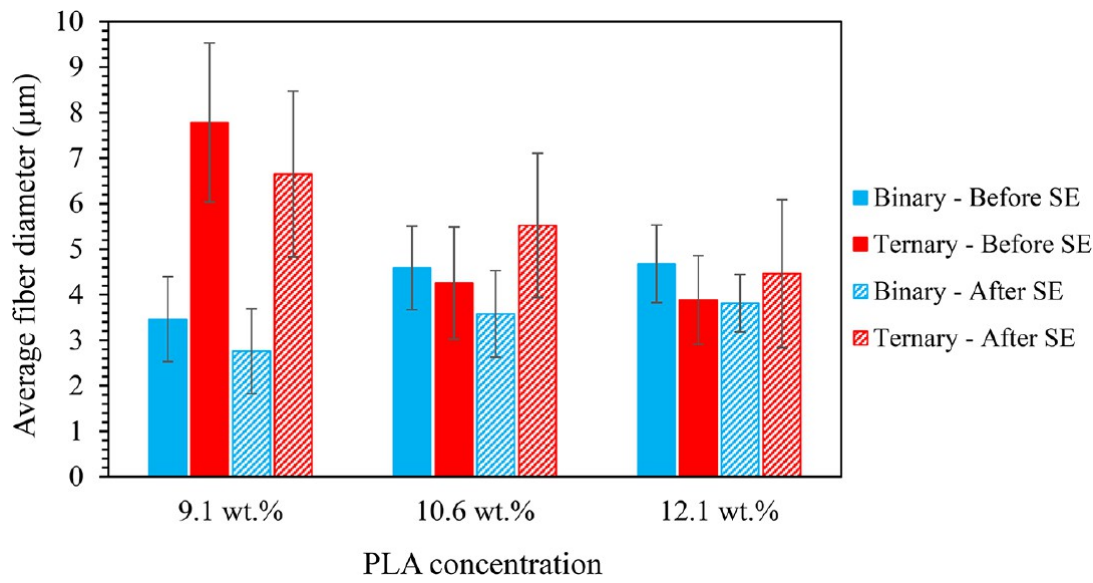

Figure 9. Average fiber diameter $(n>50)$ for each membrane electrospun from binary and ternary systems before and after solvent exchange (SE). Note that for the ternary systems, SEM images captured in the late stages of electrospinnability of each system were used, as presented in Figure 7. Error bars represent standard deviation.

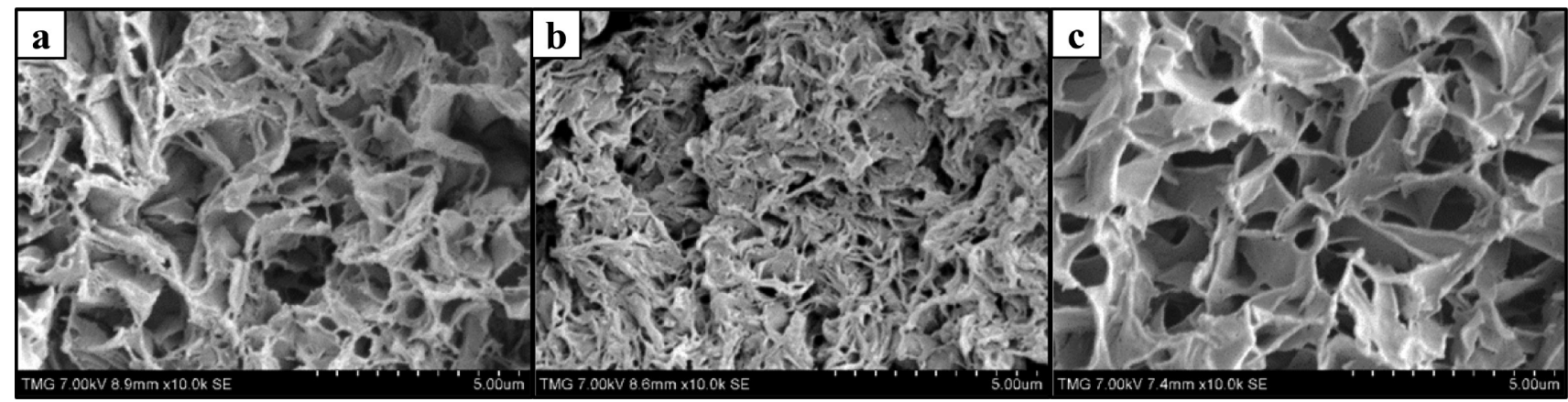

Figure 10. SEM images of the PLA foams produced from ternary systems with PLA concentrations of 9.1 wt \% (a), 10.6 wt \% (b), and 12.1 wt \% (c). Images $a$ and $b$ present the morphologies of the foams which were undergone high shrinkage during drying of the gel. ${ }^{18}$ The mean porosity of the foams correspond to images a, b, and c are $45.5 \%, 40.7 \%$, and $65.6 \%$, respectively. ${ }^{18}$

separation and the solvent exchange on the final size of the fibers.

Before solvent exchange, only the fibers produced from low PLA concentration systems (9.1 wt \%) show a significant difference between the average fiber diameter of the samples produced from binary and ternary systems. These fibers have similar surface morphology (Figures $4 \mathrm{~b}$ and $7 \mathrm{~b}$ ), but the fibers of the ternary system have porous cores (Figure 8a) contributing to the larger diameter whereas the core of the fibers of the corresponding binary system is dense (Figure $8 b$ ). Considering the standard deviations, we cannot distinguish between the diameters of the fibers produced from the 10.6 and 12.1 wt \% systems.

3.3.4. Foams. Figure 10 presents the SEM images of the NIPS-derived foams. Note that the NIPS foaming technique and the characterization of the resulting PLA foams are presented in detail in our previous study. ${ }^{18}$ The morphology and pore formation mechanisms of the foams are completely different from those of the corresponding electrospun fibers. Unlike electrospinning, in the foaming process, the ternary systems are allowed to gel quiescently (Figure 1). The solvent exchange process is an essential step of the NIPS foaming technique which prevents pore collapse during drying. Thus, the NIPS-derived foams are always produced through a process that includes a solvent exchange step. ${ }^{18}$ The solvent exchange process effectively increases the final porosity of the foams because it allows the gel to further crystallize such that it can resist capillary forces and not collapse during the subsequent air drying. ${ }^{18}$ For the electrospun fibers, the rapid evaporation, short crystallization time, applied tensile stress, and electric field affect the final structure and pore and crystal morphology. $^{20,30,31}$

3.4. Crystallization. The crystallization process of the binary and especially ternary systems is very complex since there are several factors such as solvent evaporation, hexaneinduced phase separation, applied electric field (i.e., voltage), and the tensile stress (mechanical drawing) during electrospinning, which can contribute to chain alignment. The applied electric field can enhance the crystallization kinetics of PLA, in particular, at higher temperatures and voltages. ${ }^{30}$ This is attributed to an increase in nucleation rate due to the presence of a polar repeat unit with dipoles parallel to the backbone of this macromolecule. ${ }^{30}$

DSC traces for the foam and the electrospun membranes produced from systems with $10.6 \mathrm{wt} \%$ PLA concentration are presented in Figure 11. The DSC curve of the foam exhibits only a deep melting peak, whereas the DSC curves of the membranes before solvent exchange show exothermic cold crystallization (between 75 and $105{ }^{\circ} \mathrm{C}$ ) and recrystallization (between 145 and $155{ }^{\circ} \mathrm{C}$ ) peaks. Interestingly, both cold crystallization and recrystallization peaks are eliminated as a result of solvent exchange. These observations are used later on to explain and better understand the crystallization process of these systems.

The crystallinity $\left(X_{c}\right)$ of the foams and electrospun membranes was calculated using eq $2 .^{18,23,28,32,33}$ 


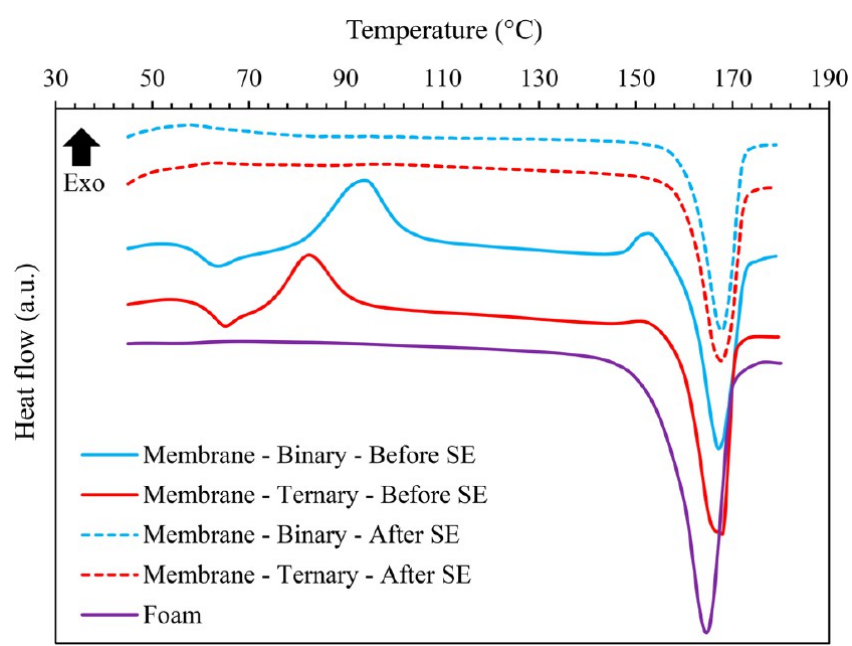

Figure 11. Typical DSC heating curves of the membranes, electrospun from binary and ternary (10.6 wt \%) systems before and after solvent exchange (SE), and the foam (10.6 wt \%). The membrane electrospun from the ternary system is produced at the late stages of its electrospinnability window. The curves are appropriately shifted vertically to avoid overlapping.

$$
X_{\mathrm{c}}=\frac{\Delta H_{\mathrm{m}}-\Delta H_{\mathrm{c}}}{\Delta H_{0}} \times 100
$$

where $\Delta H_{\mathrm{m}}$ and $\Delta H_{\mathrm{c}}$ are respectively the heat of fusion and the heat of crystallization measured from the DSC heating curves, and $\Delta H_{0}$ is the enthalpy of fusion of fully crystalline PLA (106 $\mathrm{J} / \mathrm{g}) \cdot{ }^{18,19,28}$

The crystallinity of the membranes electrospun from ternary systems at various aging times during phase separation is presented in Figure 12. According to this figure, crystallinity increases as a function of aging time during phase separation resulting in higher crystallinity for higher PLA concentrations. As the ternary systems age during their phase separation and their polymer-rich phase becomes more concentrated, more incipient crystals are formed. Thus, the final crystallinity of each

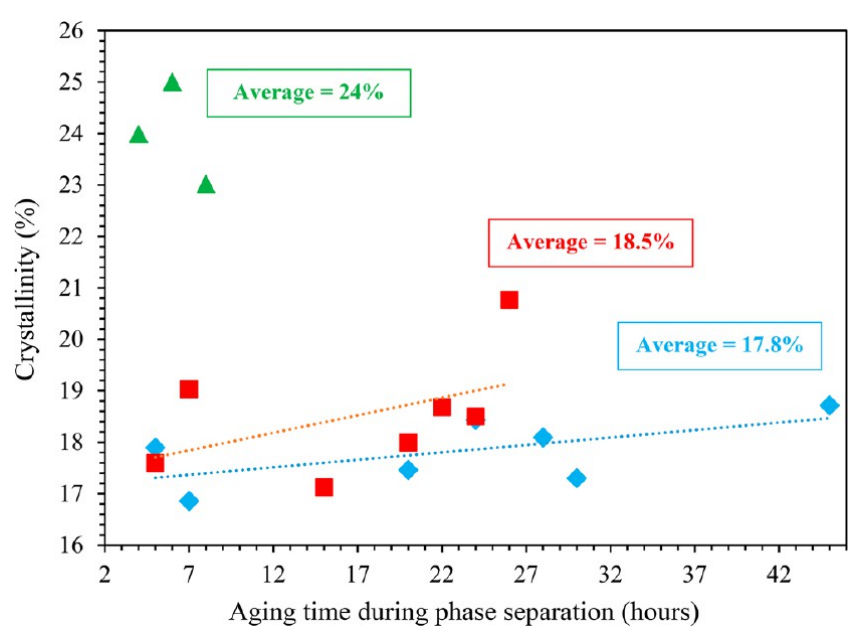

Figure 12. Crystallinity of the membranes electrospun from ternary systems with PLA concentration of $9.1 \mathrm{wt} \%(\diamond), 10.6 \mathrm{wt} \%(\boldsymbol{\square})$, and $12.1 \mathrm{wt} \%(\boldsymbol{\Delta})$ at various aging times during their phase separation. Note that these systems have different gelation times with the shortest occurring at the highest PLA concentration. The trendlines show the increasing trend in crystallinity of the membranes. membrane in Figure 12 may be related to the number density of these incipient crystals which is related to the initial polymer concentration of the system. In other words, semiordered chains of the incipient crystals within the polymer-rich phase of a phase-separated ternary system enhance the final crystallinity of its electrospun membrane.

The crystallinity values of the PLA foams and the membranes before and after solvent exchange are presented in Figure 13. Note that the crystallinity of the membranes produced from each ternary system (before solvent exchange) is obtained by averaging the crystallinity of all the membranes electrospun from that system during phase separation. The crystallinity of the membranes produced from ternary systems is on average higher than that of the binary systems which is partially due to the presence of the incipient crystals in the polymer-rich domains as explained earlier.

According to Figure 13, the crystallinity of the electrospun membranes is much lower than that of the foams due to the difference in their production routes (Figure 1). The PLA foams are produced from ternary systems which are allowed to phase separate, partially crystallize, and gel followed by a solvent exchange, all of which contribute to their higher crystallinity. ${ }^{6,18}$ On the other hand, for the membranes, the phase separation and crystallization processes are interrupted by the electrospinning process (Figure 1). The electrospinning process does not provide sufficient time for PLA crystallization, which is known to be slow, ${ }^{15,30,33-35}$ resulting in poor packing of PLA chains ${ }^{4}$ leading to low crystallinity (Figure 13). This poor chain packing is reflected in the cold crystallization exhibited by the membranes in the DSC studies (Figure 11). For both binary and ternary systems, the crystallinity of the membranes is increased after solvent exchange (Figure 13) due to crystallization during solvent exchange.

It is known that increasing temperature increases chain mobility which may lead to the cold crystallization of PLA. ${ }^{12}$ Here, we show that during solvent exchange in a nonsolvent at room temperature, cold crystallization can also occur due to the plasticization effect of the residual DCM. The solvent exchange process allows the semiordered regions to crystallize through a gradual replacement of the residual DCM with methanol in the polymer-rich domains. ${ }^{18}$ By removing the DCM in the solvent exchange process, we also prevent future chain reorganization.

Figure 14 presents the heat of cold crystallization and cold crystallization temperature of the membranes before solvent exchange. Figure 14 reveals that the average heat of cold crystallization and cold crystallization temperature for membranes produced from ternary systems are respectively $\sim 3.6 \mathrm{~J} / \mathrm{g}$ and $\sim 10{ }^{\circ} \mathrm{C}$ lower than those of the binary systems. These observations indicate that the incipient crystals within the polymer-rich domains of the ternary systems may act as nucleators and accelerate and promote crystallization during the spinning process ${ }^{12,15,34}$ and during cold crystallization in the DSC. This is also consistent with our explanation for Figure 12 that the average crystallinity of the membranes produced from the ternary system with PLA concentration of $12.1 \mathrm{wt} \%$ is higher than that of the other ternary systems due to the more numbers of incipient crystals (i.e., nucleators) promoting further crystallization.

There are four crystal forms for PLLA, $\gamma, \beta, \alpha$ (the most common form), and $\alpha^{\prime}$ (less ordered $\alpha$ ) which can be formed under suitable conditions. ${ }^{12,15,23,35}$ The $\alpha^{\prime}$ to $\alpha$ recrystallization transition is a well-known phenomenon for PLLA which normally occurs during a heating process before melting. This 


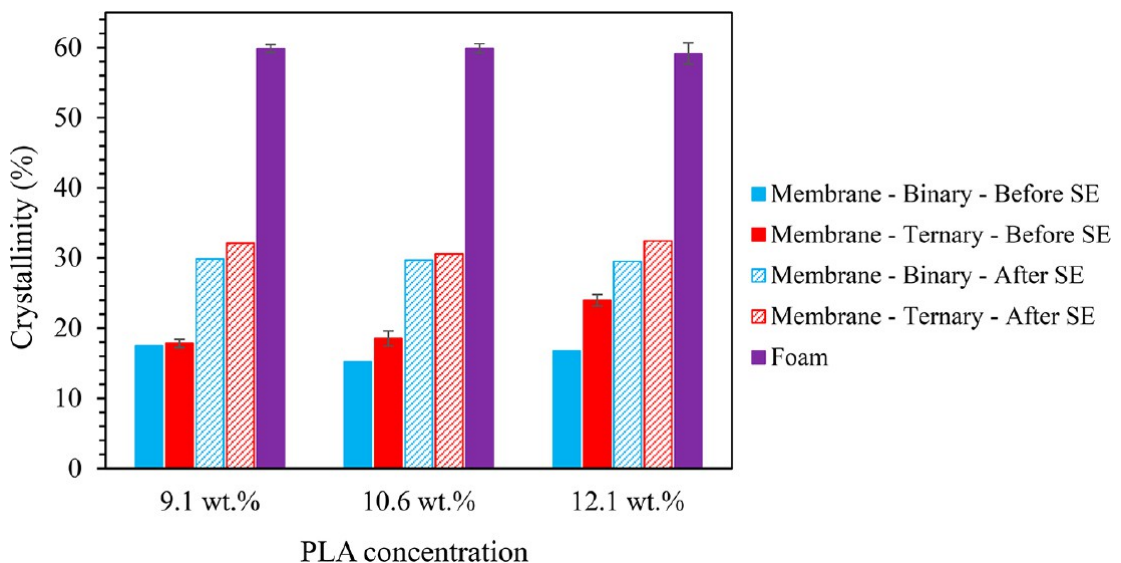

Figure 13. Crystallinity of the membranes electrospun from binary and ternary systems before and after solvent exchange (SE) and the foams. Note that the crystallinity of the membranes produced from ternary systems before SE is the average of the corresponding crystallinity values in Figure 12 . The crystallinity of the foams is obtained from ref 18 .

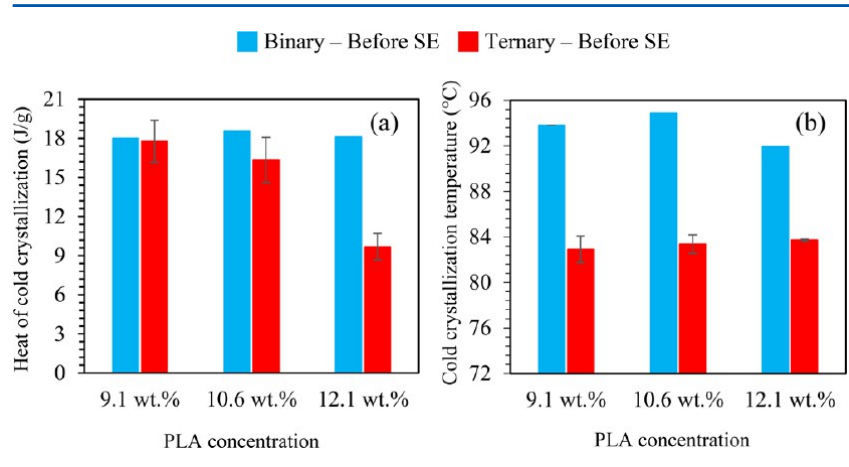

Figure 14. Heat of cold crystallization (a) and cold crystallization temperature (b) of the membranes before solvent exchange (SE). The values presented for the ternary systems are the average of the values measured for the membranes electrospun at various aging times (Figure 12). Note that cold crystallization is not observed in the DSC curves of the foams and the membranes after SE (Figure 11).

recrystallization transition is governed by a solid-solid transformation mechanism with no melting involved. ${ }^{35}$ The small exothermic peak due to $\alpha^{\prime}$ to $\alpha$ recrystallization occurs before the melting peak in the DSC curves of the membranes before solvent exchange (Figure 11). The heat of recrystallization of the membranes is presented in Figure 15. The heat of recrystallization of membranes electrospun from ternary systems is less than that of the membranes produced from binary systems (Figure 15). Additionally, as we have shown in Figure 13, the membranes electrospun from the ternary systems have a higher crystallinity (before solvent exchange). Together, this reveals that more of the perfect $\alpha$ crystals exist in aselectrospun membranes produced from ternary systems which can be again related to the nucleator role of the incipient crystals formed during phase separation as well as the lower evaporation rate (Figure 2), all of which provide suitable conditions for the formation of the more packed crystal form $(\alpha)$.

As shown in Figure 11, the recrystallization peak is not present in the DSC curves of the membranes after solvent exchange. Thus, the room temperature solvent exchange process not only allows for cold crystallization of PLA as explained but also transforms the less packed $\alpha^{\prime}$-form crystals to the perfect $\alpha$-form crystals. ${ }^{12,15,35}$

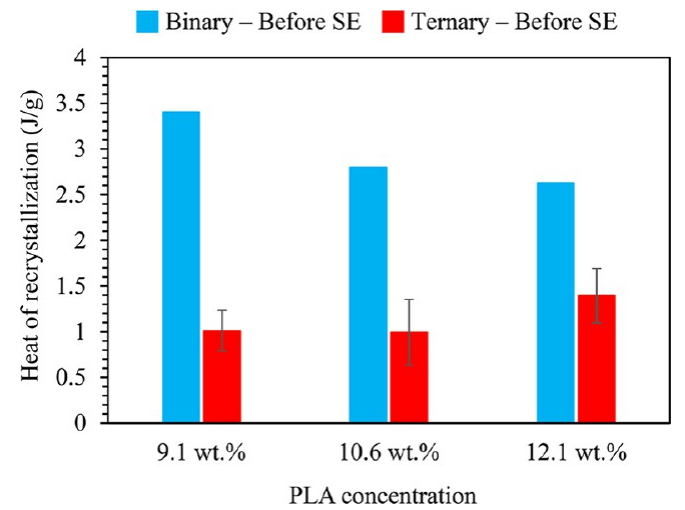

Figure 15. Heat of recrystallization of the membranes before solvent exchange (SE). The values presented for the ternary systems are the average of the values measured for the membranes electrospun at various aging times (Figure 12). Note that recrystallization is not observed in the DSC curves of the foams and the membranes after SE (Figure 11).

Similar to the morphology, the crystallinity of the aselectrospun PLA membranes produced from binary and ternary systems drastically changes during solvent exchange at room temperature in the nonsolvent methanol even though the systems contain very volatile solvents which are expected to be eliminated during the electrospinning process. These observations illuminate an important issue that regardless of the volatility of the solvent(s), as-electrospun membranes produced from solutions may not maintain their pore morphology and/or crystallinity during subsequent applications such as filtration where the membranes are in contact with a fluid. Depending on several factors discussed in this paper, such as the polymersolvent(s) interaction parameter, an appropriate aging process may be essential after electrospinning to improve the in-service performance of the membranes.

\section{CONCLUSIONS}

In this study, we produced and characterized PLA membranes electrospun from PLA-DCM binary and PLA-DCM-hexane ternary systems under constant ambient and processing parameters. We presented a rheological model that can identify the initiation of the electrospinnability window for the ternary systems during which viscoelastic properties of the system allow 
for the formation of a more stable, continuous jet. According to this model, the electrospinnability window of the ternary systems studied here starts within at most $10 \%$ of their induction time of crystallization. Thus, the electrospinnability of phase separating ternary systems may be related to the formation of incipient crystals within their polymer-rich phase. The DSC results showed that the semiordered chains of these incipient crystals may act as nucleators for further crystallization leading to higher crystallinity and more packed $\alpha$ crystals for the membranes produced from ternary systems compared to those of the binary systems. Also, crystallinity of the membranes increases over aging time during phase separation of each ternary system as well as with the PLA concentration.

The fibers electrospun from binary systems are porous on the surface and dense in the core whereas the fibers from the ternary systems have a very rough surface and a highly porous core. We also showed that the surface roughness is a result of surface porosity coarsening at a critical polymer concentration. The surface porosity of the fibers of the binary systems is formed as a result of a combination of breath figures, TIPS, and/or humidity-induced phase separation mechanisms. For the ternary systems, the hexane-induced phase separation has the predominant role in the formation of the surface roughness and core porosity. A combination of spinodal and nucleation and growth phase separation controls the pore formation in the ternary system.

Despite the high volatility of hexane and especially DCM as well as the rapid increase in specific surface area of the system during electrospinning, there remains residual solvent in the fibers that can affect their properties after deposition. As a result, the surface porosity of the fibers electrospun from binary solutions was decreased after immersing in a nonsolvent (methanol) at room temperature. Except for low PLA concentration, the rough surface texture of the fibers electrospun from ternary systems was not affected by the solvent exchange. This highlights the importance of the polymersolvent interaction parameter in such a process which is mostly overlooked in the literature compared to the other parameters such as the volatility of solvent(s). We also showed that the residual solvent(s) can potentially cause even a more significant impact on the crystallinity of the electrospun fibers. The crystallinity of the membranes produced from binary and ternary systems are respectively increased by $79.9 \%$ and $57.6 \%$ on average, after solvent exchange in methanol. The gradual diffusion of residual DCM into the methanol provides the chains with sufficient time to order and further crystallize. The DSC results revealed that the membranes cold crystallize and recrystallize ( $\alpha^{\prime}$ to $\alpha$ ) during this solvent exchange.

These results and observations illustrate an important issue that has not been properly addressed in the literature: the surface morphology and crystallinity of as-electrospun PLA membranes can change during their subsequent characterization and application influencing their in-service performance. It is very likely that a similar morphological and crystallinity change would occur after solvent exchange for other systems containing a polymer and a good solvent which have a low interaction parameter (i.e., small $\left|\delta_{\text {polymer }}-\delta_{\text {solvent }}\right|$ ) and also form persistent hydrogen bonds.

\section{ASSOCIATED CONTENT}

\section{Supporting Information}

The Supporting Information is available free of charge on the ACS Publications website at DOI: 10.1021/acs.macromol.8b00083.

Experimentally developed PLA-DCM-hexane phase diagram; rod climbing phenomenon; complex viscosity as a function of time for the binary solution with $10.6 \mathrm{wt}$ $\%$ PLA; SEM images of higher magnifications from the porous microbead (Figure 6a) (PDF)

\section{AUTHOR INFORMATION}

\section{Corresponding Author}

*Tel +1 (514) 9930028; e-mail e.rezabeigi@gmail.com (E.R.). ORCID

Ehsan Rezabeigi: 0000-0003-2968-6045

Paula M. Wood-Adams: 0000-0003-1562-3924

\section{Notes}

The authors declare no competing financial interest.

\section{ACKNOWLEDGMENTS}

Funding was provided by NSERC, École de Technologie Supérieure, and Concordia University. The authors gratefully thank Ms. Marwa Sta and Dr. Changsheng Wang for their help with some of the electrospinning and rheological experiments, respectively.

\section{REFERENCES}

(1) Megelski, S.; Stephens, J. S.; Chase, D. B.; Rabolt, J. F. Micro- and Nanostructured Surface Morphology on Electrospun Polymer Fibers. Macromolecules 2002, 35, 8456-8466.

(2) Gupta, A.; Saquing, C. D.; Afshari, M.; Tonelli, A. E.; Khan, S. A.; Kotek, R. Porous Nylon-6 Fibers via a Novel Salt-Induced Electrospinning Method. Macromolecules 2009, 42, 709-715.

(3) Kim, J. F.; Kim, J. H.; Lee, Y. M.; Drioli, E. Thermally Induced Phase Separation and Electrospinning Methods for Emerging Membrane Applications: A Review. AIChE J. 2016, 62, 461-490.

(4) Wang, B.; Li, B.; Xiong, J.; Li, C. Y. Hierarchically Ordered Polymer Nanofibers via Electrospinning and Controlled Polymer Crystallization. Macromolecules 2008, 41, 9516-9521.

(5) Chen, P.-Y.; Tung, S.-H. One-Step Electrospinning To Produce Nonsolvent-Induced Macroporous Fibers with Ultrahigh Oil Adsorption Capability. Macromolecules 2017, 50, 2528-2534.

(6) Bognitzki, M.; Czado, W.; Frese, T.; Schaper, A.; Hellwig, M.; Steinhart, M.; Greiner, A.; Wendorff, J. H. Nanostructured Fibers via Electrospinning. Adv. Mater. 2001, 13, 70-72.

(7) Shenoy, S. L.; Bates, W. D.; Frisch, H. L.; Wnek, G. E. Role of chain entanglements on fiber formation during electrospinning of polymer solutions: good solvent, non-specific polymer-polymer interaction limit. Polymer 2005, 46, 3372-3384.

(8) Casper, C. L.; Stephens, J. S.; Tassi, N. G.; Chase, D. B.; Rabolt, J. F. Controlling Surface Morphology of Electrospun Polystyrene Fibers: Effect of Humidity and Molecular Weight in the Electrospinning Process. Macromolecules 2004, 37, 573-578.

(9) Kongkhlang, T.; Kotaki, M.; Kousaka, Y.; Umemura, T.; Nakaya, D.; Chirachanchai, S. Electrospun Polyoxymethylene: Spinning Conditions and Its Consequent Nanoporous Nanofiber. Macromolecules 2008, 41, 4746-4752.

(10) Mascia, L.; Su, R.; Clarke, J.; Lou, Y.; Mele, E. Fibres from blends of epoxidized natural rubber and polylactic acid by the electrospinning process: Compatibilization and surface texture. Eur. Polym. J. 2017, 87, 241-254. 
(11) Luo, C. J.; Stride, E.; Edirisinghe, M. Mapping the Influence of Solubility and Dielectric Constant on Electrospinning Polycaprolactone Solutions. Macromolecules 2012, 45, 4669-4680.

(12) Rezabeigi, E.; Sta, M.; Swain, M.; McDonald, J.; Demarquette, N. R.; Drew, R. A. L.; Wood-Adams, P. M. Electrospinning of porous polylactic acid fibers during nonsolvent induced phase separation. $J$. Appl. Polym. Sci. 2017, 44862.

(13) Viswanath, V.; Maity, S.; Bochinski, J. R.; Clarke, L. I.; Gorga, R. E. Enhanced Crystallinity of Polymer Nanofibers without Loss of Nanofibrous Morphology via Heterogeneous Photothermal Annealing. Macromolecules 2016, 49, 9484-9492.

(14) Qi, Z.; Yu, H.; Chen, Y.; Zhu, M. Highly porous fibers prepared by electrospinning a ternary system of nonsolvent/solvent/poly(Llactic acid). Mater. Lett. 2009, 63, 415-418.

(15) Xiao, H.; Yang, L.; Ren, X.; Jiang, T.; Yeh, J.-T. Kinetics and Crystal Structure of Poly(lactic acid) Crystallized Nonisothermally: Effect of Plasticizer and Nucleating Agent. Polym. Compos. 2010, 31, 2057-2068.

(16) Katsogiannis, K. A. G.; Vladisavljevic, G. T.; Georgiadou, S. Porous electrospun polycaprolactone (PCL) fibres by phase separation. Eur. Polym. J. 2015, 69, 284-295.

(17) Rezabeigi, E.; Wood-Adams, P. M.; Drew, R. A. L. Isothermal ternary phase diagram of the polylactic acid-dichloromethane-hexane system. Polymer 2014, 55, 3100-3106.

(18) Rezabeigi, E.; Wood-Adams, P. M.; Drew, R. A. L. Production of porous polylactic acid monoliths via nonsolvent induced phase separation. Polymer 2014, 55, 6743-6753.

(19) Rezabeigi, E.; Drew, R. A. L.; Wood-Adams, P. M. Highly Porous Polymer Structures Fabricated via Rapid Precipitation from Ternary Systems. Ind. Eng. Chem. Res. 2017, 56, 11451-11459.

(20) Fashandi, H.; Ghomi, A. Interplay of Phase Separation and Physical Gelation in Morphology Evolution within Nanoporous Fibers Electrospun at High Humidity Atmosphere. Ind. Eng. Chem. Res. 2015, 54, 240-253.

(21) Di Luccio, M.; Nobrega, R.; Borges, C. Microporous anisotropic phase inversion membranes from bisphenol-A polycarbonate: study of a ternary system. Polymer 2000, 41, 4309-4315.

(22) Nayani, K.; Katepalli, H.; Sharma, C. S.; Sharma, A.; Patil, S.; Venkataraghavan, R. Electrospinning Combined with NonsolventInduced Phase Separation To Fabricate Highly Porous and Hollow Submicrometer Polymer Fibers. Ind. Eng. Chem. Res. 2012, 51, 17611766.

(23) He, L.; Zhang, Y.; Zeng, X.; Quan, D.; Liao, S.; Zeng, Y.; Lu, J.; Ramakrishna, S. Fabrication and characterization of poly(L-lactic acid) 3D nanofibrous scaffolds with controlled architecture by liquid-liquid phase separation from a ternary polymer-solvent system. Polymer 2009, 50, 4128-4138.

(24) Yu, X.; Xiang, H.; Long, Y.; Zhao, N.; Zhang, X.; Xu, J. Preparation of porous polyacrylonitrile fibers by electrospinning a ternary system of PAN/DMF/ $\mathrm{H}_{2} \mathrm{O}$. Mater. Lett. 2010, 64, 2407-2409.

(25) Tang, C.; Saquing, C. D.; Harding, J. R.; Khan, S. A. In Situ Cross-Linking of Electrospun Poly(vinyl alcohol) Nanofibers. Macromolecules 2010, 43, 630-637.

(26) Sperling, L. H. Introduction to Physical Polymer Science; John Wiley \& Sons, Inc.: Hoboken, NJ, 2006.

(27) Chaeichian, S.; Wood-Adams, P. M.; Hoa, S. In situ polymerization of polyester-based hybrid systems for the preparation of clay nanocomposites. Polymer 2013, 54, 1512-1523.

(28) Rezabeigi, E.; Wood-Adams, P. M.; Drew, R. A. L. Crystallization of Polylactic Acid under in Situ Deformation during Nonsolvent-Induced Phase Separation. J. Polym. Sci., Part B: Polym. Phys. 2017, 55, 1055-1062.

(29) Koski, A.; Yim, K.; Shivkumar, S. Effect of molecular weight on fibrous PVA produced by electrospinning. Mater. Lett. 2004, 58, 493497.

(30) Yuryev, Y.; Wood-Adams, P. M. Crystallization of Poly(L -/ D -Lactide) in the Presence of Electric Fields. Macromol. Chem. Phys. 2012, 213, 635-642.
(31) Lee, K.-H.; Snively, C. M.; Givens, S.; Chase, D. B.; Rabolt, J. F. Time-Dependent Transformation of an Electrospun Isotactic Poly(1butene) Fibrous Membrane. Macromolecules 2007, 40, 2590-2595.

(32) Kong, Y.; Hay, J. N. The measurement of the crystallinity of polymers by DSC. Polymer 2002, 43, 3873-3878.

(33) Suksut, B.; Deeprasertkul, C. Effect of Nucleating Agents on Physical Properties of Poly(lactic acid) and Its Blend with Natural Rubber. J. Polym. Environ. 2011, 19, 288-296.

(34) Ni, C.; Luo, R.; Xu, K.; Chen, G.-Q. Thermal and Crystallinity Property Studies of Poly (L-Lactic Acid) Blended with Oligomers of 3Hydroxybutyrate or Dendrimers of Hydroxyalkanoic Acids. J. Appl. Polym. Sci. 2009, 111, 1720-1727.

(35) Shen, C.; Wang, Y.; Li, M.; Hu, D. Crystal Modifications and Multiple Melting Behavior of Poly(L-lactic acid-co-D-lactic acid). J. Polym. Sci., Part B: Polym. Phys. 2011, 49, 409-413. 